\title{
EPIDEMIOLOGIA DA LEISHMANIOSE TEGUMENTAR AMERICANA E SUAS RELAÇŌES COM A LAVOURA E O GARIMPO, EM LOCALIDADE DO ESTADO DA BAHIA (BRASIL)
}

\author{
Maria Inês Costa Dourado* \\ Ceci Vilar Noronha* \\ Neusa Alcantara** \\ Maria Yuri T. Ichihara*** \\ Sebastião Loureiro*
}

DOURADO, M.I.C. et al. Epidemiologia da leishmaniose tegumentar americana e suas relaçōes com a lavoura e o garimpo, em localidade do Estado da Bahia (Brasil). Rev. Saúde públ., S.Paulo, 23:2-8, 1989.

\begin{abstract}
RESUMO: Realizou-se um estudo sobre a associação entre in fecção leishmaniótica e a ocupaçăo dos indivíduos em Lençóis-Bahia (Brasil). Foram utilizados um questionário com variáveis biológicas, sociais e económicas e o teste de Montenegro. Houve captura de flebotomineos em locais peri e intra-domiciliares. Encontrou-se maior prevalência da infecção no grupo ocupacional de lavradores/garimpeiros em comparaçăo ds demais ocupaçōes. Explica-se esse resultado em razào da dupla exposição dos lavradores/garimpeiros à leishmaniose tegumentar americana, em sua área de moradia e local de trabalho.
\end{abstract}

DESCRITORES: Leishmania mucocutânea, incidência. Ocupação. Flebotomíneos. Ecologia de vetores.

\section{INTRODUÇÃO}

A leishmaniose tegumentar americana (LTA) é uma enfermidade que apresenta cadeia de transmissão complexa, estando sujeita numa mesma região a diversos determinantes tais como: desequilíbrio ecológico produzido pela invasão do homem aos nichos naturais da doença, variações sazonais e suceptibilidade da populaçãos,17.

No município de Lençóis - Bahia, entre junho de 1985 e fevereiro de 1986, foram notificados pela Superintendência de Campanhas de Saúde Pública (SUCAM), 143 casos de LTA ${ }^{25}$. Pela distribuição dos casos notificados, a transmissão da enfermidade não ocorria exclusivamente na cidade de Lençóis incluindo mais quatro fazendas vizinhas. Contudo, $90 \%$ dos casos ocorreram entre os habitantes da sede municipal.

Do início do surto até agosto de 1985, registrou-se um total de 18 casos. A partir desse período, há um aumento de casos por mês tendo maior expressão (33 casos) em novembro. No ano de 1986 o surto começou a decrescer passando de 29 casos (dezembro de 1985) para 19 em janeiro e $6 \mathrm{em}$ fevereiro ${ }^{25}$.
Os primeiros estudos de LTA, no país ${ }^{20}$, identificaram um padrão de transmissão fundamentado nos contatos do homem com o meio ambiente silvestre, onde a enfermidade pré-existe sob a forma de enzoose. Assim, os grupos humanos mais expostos a contrair esta enfermidade seriam constituídos por indivíduos que trabalham em atividades extrativas vegetais ou minerais e aqueles que se ocupam da construção de obras de engenharia civil desenvolvidas em zonas florestais. As atividades agrícolas, principalmente por sua característica de expansão com a incorporação de novas terras, através dos desmatamentos, também propiciaram uma maior prevalência da doença entre os lavradores.

Desta forma, é possível estabelecer a partir da literatura especializada $1,2,3,4,13,19,23$, uma associação entre trabalho agrícola e LTA. Entretanto, a dinâmica do desenvolvimento econômico e suas implicações, nas condições de vida e trabalho no campo, impelem para o questionamento da veracidade dessa associação em determinadas áreas do território brasileiro.

O objetivo específico definido para a presente investigação foi medir a prevalência da infecção por Leishmania sp., analisando-a em relação à ocupação dos indivíduos.

- Departamento de Medicina Preventiva da Universidade Federal da Bahia - Rua Padre Feijó, 29 - 4. andar - 40000 - Salvador, BA - Brasil.

*** Instituto de Ciências da Saúde da Universidade Federal da Bahia -- Rua Padre Feijó, 29 - 40000 - Salvador, BA Brasil.

*** Superintendência de Campanhas de Saúde Pública (SUCAM/Bahia) - 40000 - Salvador, BA - Brasil. 


\section{MATERIAL E MÉTODO}

O município de Lençóis situa-se a 409,3 km de Salvador, Bahia, e pertence a microrregião da Chapada Diamantina. A população total do município é de 3.254 habitantes. A altitude é de $439 \mathrm{~m}$ com uma pluviosidade média anual de $1.350 \mathrm{~mm}$. O clima é úmido, tendo uma temperatura média anual de $22,1^{\circ} \mathrm{C}$. O relêvo é composto por piemontes, patamares interfluviais, chapadões sedimentares e serras da cadeia do Espinhaço. A vegetação do município compreende caatingas e refúgios ecológicos. Em tôrno da cidade de Lençóis, a vegetação é classificada como floresta subperenefolia, ou seja, a vegetação torna-se rarefeita em determinada época do ano, voltando a se recompor em outro periodo 24 .

Em maio de 1986, quando esta investigação epidemiológica teve início, a SUCAM vinha desenvolvendo ações de controle dos vetores, inquérito sorológico para os cães e os casos humanos estavam sendo tratados no serviço de saúde local. O grupo de casos compreendia 25 menores de 15 anos, sendo 12 do sexo masculino e 13 do sexo feminino, cuja distribuição na cidade de Lençóis apontava para uma concentração de casos em duas áreas periféricas em que a proximidade física das habitações com a mata era maior ${ }^{25}$.

No inquérito epidemiológico, foram utilizados dois instrumentos: a) o teste alérgico de Montenegro, usando o antígeno produzido pela Fundação de Amparo à Pesquisa Parasitológica da Universidade Federal de Minas Gerais. O antígeno era inoculado na dose de $0,1 \mathrm{ml}$, intradermicamente na face anterior do antebraço direito. A leitura das reações eram feitas com $48 \mathrm{~h}$ e se considerou positiva as indurações com diâmetros iguais ou superiores a $5 \mathrm{~mm}$; b) um questionário com variáveis biológicas, sociais e econômicas.

Simultaneamente realizou-se um inquérito entomológico nas áreas do estudo, num período de 30 dias, quando empreendeu-se 38 capturas de flebotomíneos, utilizando-se armadilha luminosa (no horário de 18 às 6 hs do dia seguinte) e capturador manual (no horário das 18 às $21 \mathrm{hs}$ ).

Para a realização do inquérito, a cidade de Lençóis foi subdividida em três áreas: duas periféricas e uma central (Fig.). As três áreas do estudo foram definidas a partir das suas características físicas e populacionais. A área l representa a área mais distante do centro comercial, localiza-se na outra margem do rio Lençóis, sua conformação de relêvo menos acidentado a torna uma área típica de expansão da cidade, onde predominam habitaçōes precárias, erguidas pe- los novos moradores que se incorporaram à cidade ao abandonar o campo; as ruas não são pavimentadas e a falta de condições adequadas de saneamento são evidentes. As características da área II são: a proximidade maior ao centro comercial da cidade, as habitações simples e antigas, os moradores com um tempo médio maior de residência na área, e a presença freqüente de árvores frutíferas nos quintais, o que pode ocasionar uma densidade maior de insetos na área. A área III é parcialmente comercial e residencial, representa o local de moradia das famílias de maiores rendas e as condições gerais de saneamento são mais apropriadas.

Para estudar a associação entre ocupação e LTA, foram considerados como população de estudo, os individuos com idade igual ou superior a 15 anos que constitui o grupo de trabalhadores por excelência numa população.

O levantamento de dados foi realizado entre maio e junho de 1986 por entrevistadores treinados que se dirigiam aos domicílios. Cada área foi trabalhada de forma censitária, percorrendo-se todas as casas habitadas.

Os problemas que dificultaram a cobertura total da população nas áreas estudadas foram recusas, viagem dos indivíduos, casos de doenças e ausência prolongada do domicílio por motivo de trabalho (afastamento de 8 a 11 dias). As recusas foram também relacionadas aos efeitos da reação intradérmica tais como a formação de edema e flictema no local de aplicação do antígeno.

O desenho adotado pela investigação foi o de prevalência por áreas específicas da cidade (áreas $1,11,111)$. A variável dependente do estudo foi a presença de infecção por Leishmania $s p$. na população. Área de residência, sexo, idade e ocupação foram tomadas como variáveis independentes capazes de influir na prevalência da infecção.

Para a análise, a ocupação de garimpeiro, comum entre os moradores de Lençóis, foi considerada com o mesmo status de risco que a ocupação de lavrador. Aceita-se, portanto, que os garimpeiros desempenham sua atividade de trabalho em condições muito próximas às dos lavradores, em contato direto com a natureza, atravessando, por vezes ou diariamente, a mata para chegar ao seu local de trabalho, permanecendo por muitas horas em ambiente propicio às picadas de insetos, pois, trata-se de trabalho desenvolvido ao ar livre.

Após a crítica dos dados, foi organizado o arquivo para a analise, utilizando-se como procedimentos estatísticos a frequência, a percentagem e o teste do qui-quadrado. 


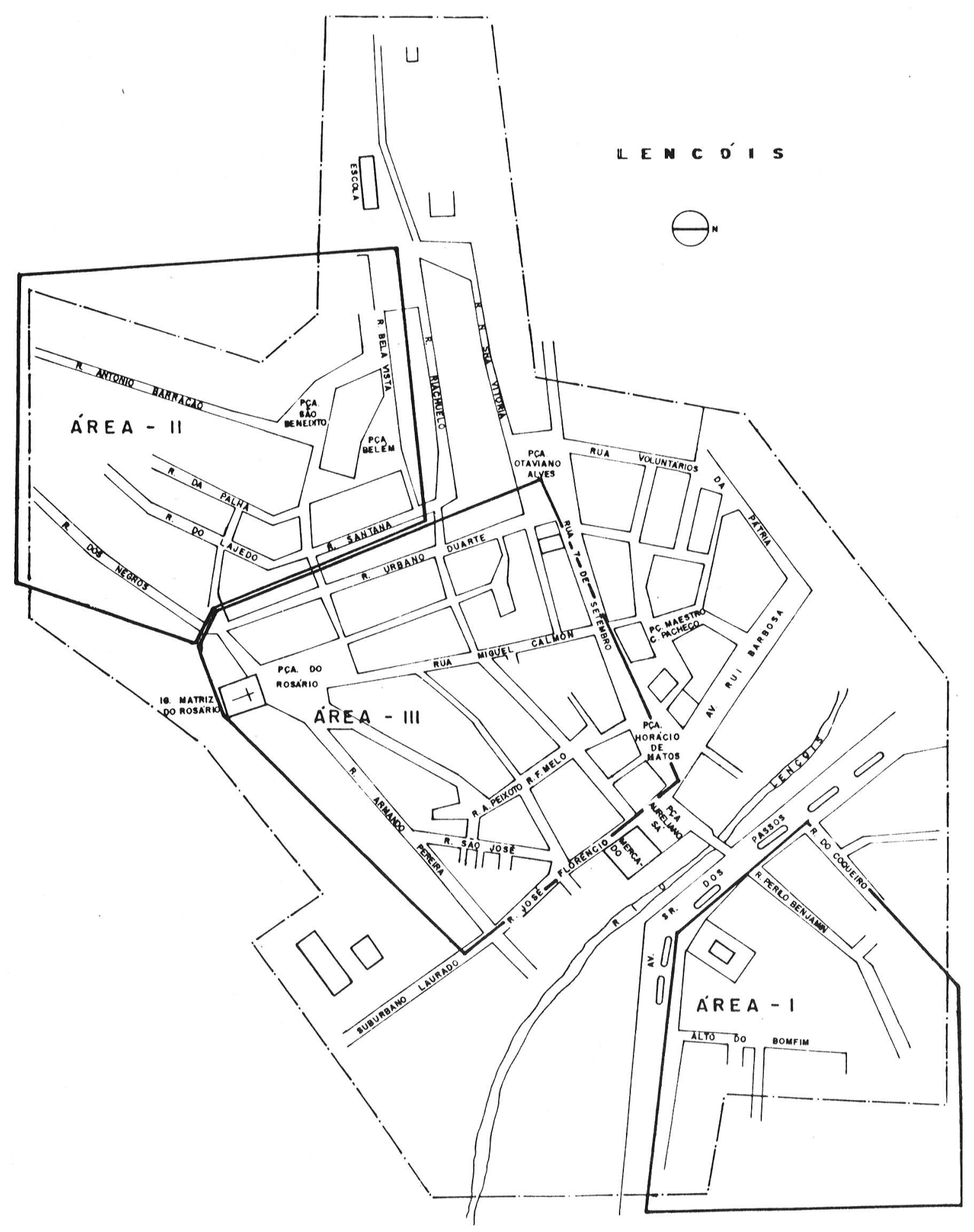

Figura - Mapa do Município de Lençóis (Bahia), dividido em três áreas, I, II (periferias) e III (central). 


\section{RESULTADOS}

O total da população estudada, com idade igual ou superior a 15 anos, nas áreas delimitadas previamente, correspondeu a 466 individuos.

A taxa de infecção por Leishmania observada em Lençóis foi de 43,3\% para o conjunto de indivíduos pesquisados. Essa taxa de infecção apresentou um comportamento diferenciado em relação às áreas, sendo decrescente a taxa de infecção quando se passa da área $I(51,1 \%)$ para a área III $(33,1 \%)$. Destaca-se assim, que as duas áreas periféricas da cidade (I e II) apresentaram taxas de infecção maiores que a área central da cidade sendo essas diferenças estatísticamente significativas (Tabela I).

\section{TABELA 1}

Resultados da intradermorreação de Montenegro segundo a área de residência. Lençóis - Bahia, 1986.

\begin{tabular}{lccc}
\hline & Examinados & \multicolumn{2}{c}{ Positivos } \\
Área de & $n ?$ & $n ?$ & $\%$ \\
Residência & $\mathrm{n}$ & 68 & 51,1 \\
\hline Área I & 133 & 93 & 44,5 \\
Área II & 209 & 41 & 33,1 \\
Área III & 124 & 202 & 43,3 \\
\hline Total & 466 & & 402 \\
\hline
\end{tabular}

$\mathrm{X}^{2}=8,73 ; \mathrm{p}=0,01$

Em relação à área I houve quatro capturas peridomiciliares e uma intra-domiciliar. Nesta área foram obtidos 145 flebotomíneos, assim distribuídos: Lutzomyia whitmani (70 e 10\%); Lutzomyia longipalpis (1080 e 6९); Lutzomyia evandroi $\left(110^{\circ}\right)$; Lutzomyia sp. $\left(40^{\circ}\right)$. Na residência onde houve a captura intradomiciliar positiva, cinco individuos contraíram a enfermidade. Os casos compreendiam crianças de um ano; 3 anos; 9, 12 e 13 anos.

Verifica-se no grupo estudado que os homens são mais infectados $(57,3 \%)$ que as mulheres $(34,2 \%)$, diferença essa estatísticamente significante. $\mathrm{O}$ fato da infecção atingir mais os homens pode estar hipoteticamente relacionado à maior proporção de homens inseridos nas atividades da produção econômica (Tabela 2).

Em Lençóis as taxas de infecção leishmaniótica por faixas etárias deixa perceber uma associação estatísticamente significante entre essas duas variáveis. Há um crescimento contínuo da taxa de infecção quando se observa as faixas etárias mais jovens até a faixa de 55 a 74 anos. $\mathrm{Na}$ faixa etária mais avançada, acima dos 74 anos, há um decréscimo da infeç̧ão que pode
TABELA 2

Resultados da intradermorreação de Montenegro segundo o sexo. Lençóis - Bahia, 1986.

\begin{tabular}{lccc}
\hline & Examinados & \multicolumn{2}{c}{ Positivos } \\
Sexo & $\mathrm{n}$ \% & $\mathrm{n}$ \% & $\%$ \\
\hline Masculino & 185 & 106 & 57,3 \\
Feminino & 281 & 96 & 34,2 \\
\hline Total & 466 & 202 & 43,3 \\
\hline
\end{tabular}

$\mathrm{X}^{2}=23,4 ; p=0,00001$

ser atribuido ao fato já observado de que os indivíduos idosos tendem a diminuir suas respostas imunitárias a qualquer tipo de antígeno e que, particularmente em relação à infecção por Leishmania pode ocorrer a negativação da resposta ao antígeno intradérmico com o decorrer dos anos pós-infecçãó.

O pico da infecção situa-se no grupo etário de 55 a 74 anos que corresponde a uma prevalência de $56,3 \%$ (Tabela 3).

TABELA 3

Resultados da intradermorreação de Montenegro segundo idade. Lençóis - Bahia, 1986.

\begin{tabular}{|c|c|c|c|}
\hline \multirow{2}{*}{$\begin{array}{l}\text { Idade } \\
\text { (anos) }\end{array}$} & \multirow{2}{*}{$\begin{array}{c}\text { Examinados } \\
n !\end{array}$} & \multicolumn{2}{|c|}{ Positivos } \\
\hline & & no & $\%$ \\
\hline $\begin{array}{c}15-34 \\
35-54 \\
55-74 \\
+75\end{array}$ & $\begin{array}{r}223 \\
114 \\
96 \\
33\end{array}$ & $\begin{array}{l}84 \\
51 \\
54 \\
13\end{array}$ & $\begin{array}{l}37,7 \\
44,7 \\
56,3 \\
39,4\end{array}$ \\
\hline Total & 466 & 202 & 43,3 \\
\hline
\end{tabular}

$\mathrm{x}^{2}=9,74 ; \mathrm{p}=0,02$

Observando a relação entre infecção por Leishmania e a ocupação desempenhada pelos indivíduos, vê-se que os lavradores e garimpeiros são mais infectados $(66,8 \%)$ do que os indivíduos que exercem outras ocupações, inclusive as donas de casa $(39,0 \%)$. Esta diferença entre as taxas de infecção dos dois grupos foi estatísticamente significante (Tabela 4).

$\mathrm{Na}$ distribuição da resposta ao teste intradérmico de Montenegro por ocupação e área residencial, vê-se que a diferenciação das taxas de infecção por grupos ocupacionais foi estatisticamente significante apenas na área II. Na I, embora haja proporção maior de infectados no grupo de lavradores e garimpeiros $(67,9 \%)$, há também proporção alta de infectados $(47,4 \%)$ no outro grupo ocupacional, não sendo, portanto, tais diferenças estatísticamente signifi- 
TABELA 4

Resultados da intradermorreação de Montenegro segundo a ocupação. Lençóis - Bahia, 1986.

\begin{tabular}{|c|c|c|c|}
\hline \multirow[b]{2}{*}{ Ocupação } & \multirow{2}{*}{$\begin{array}{c}\text { Examinados } \\
n ?\end{array}$} & \multicolumn{2}{|c|}{ Positivos } \\
\hline & & n! & $\%$ \\
\hline $\begin{array}{l}\text { Lavrador e/ou } \\
\text { garimpeiro }\end{array}$ & 79 & 52 & 65,8 \\
\hline $\begin{array}{l}\text { Outras } \\
\text { ocupaçōes }\end{array}$ & 351 & 137 & 39,0 \\
\hline Total & $430^{*}$ & 189 & 44,0 \\
\hline
\end{tabular}

$\mathrm{X}^{2}=17,7 ; \mathrm{p}=0,00001$

* Faltam 36 informaçōes ocupacionais

cantes. Vê-se também que a área III corresponde àquela em que os lavradores e garimpeiros se encontram em menor número, não se observando, assim, diferenças significativas da infecção por grupo ocupacional. Esses resultados sugerem que a associação entre ocupação e LTA está sujeita a condicionantes outros do modo de vida da população e ao padrão de transmissão da enfermidade (Tabela 5).
O estudo da distribuição conjunta das variáveis sexo e infecção, por área residencial, coloca em evidência que a associação entre sexo e LTA não é verdadeira na área 1 . Assim, nesta área as mulheres estão tão infectadas quanto os homens. As proporções são de 50,0\% para o sexo feminino e $52,7 \%$ para o masculino. Este resultado é divergente em relação às demais áreas (Tabela 6).

\section{DISCUSSÃO}

O padrão de transmissão da LTA em Lençóis pode ser caracterizado na área I como peridomiciliar e domiciliar considerando: a presença de homens e mulheres com a mesma taxa de infecção; a existência de doentes de todas as faixas etárias inclusive crianças de 1 a 2 anos; a proximidade física maior das habitações da área em relação à mata; a não diferenciação das taxas de infecção de lavradores e garimpeiros em relação às demais ocupações e a presença de flebotomíneos, inclusive no interior de uma residência.

TABELA 5

Resultados da intradermorreação de Montenegro segundo a ocupação e área residencial. Lençóis - Bahia, 1986.

\begin{tabular}{|c|c|c|c|c|c|c|c|}
\hline \multirow{3}{*}{ Área } & \multicolumn{3}{|c|}{ Lavrador/Garimpeiro } & \multicolumn{3}{|c|}{ Outras Ocupações } & \multirow{3}{*}{$\mathrm{x}^{2}$} \\
\hline & \multirow{2}{*}{$\begin{array}{c}\text { Examinados } \\
\text { n! }\end{array}$} & \multicolumn{2}{|c|}{ Positivos } & \multirow{2}{*}{$\begin{array}{c}\text { Examinados } \\
\text { n? }\end{array}$} & \multicolumn{2}{|c|}{ Positivos } & \\
\hline & & n! & $\%$ & & n? & $\%$ & \\
\hline Ärea I & 28 & 19 & 67,9 & 97 & 46 & 47,4 & $p=0,09$ \\
\hline Área II & 42 & 29 & 69,0 & 159 & 62 & 39,0 & $p=0,0009$ \\
\hline Área III & 9 & 4 & 44,4 & 95 & 29 & 30,5 & $\begin{array}{c}0,23 \\
\mathrm{p}>0,05\end{array}$ \\
\hline
\end{tabular}

TABELA 6

Resultados da intradermorreação de Montenegro segundo o sexo e área residencial. Lençóis - Bahia, 1986.

\begin{tabular}{|c|c|c|c|c|c|c|c|}
\hline \multirow[b]{3}{*}{ Área } & \multicolumn{3}{|c|}{ Masculino } & \multicolumn{3}{|c|}{ Feminino } & \multirow{3}{*}{$x^{2}$} \\
\hline & \multirow{2}{*}{$\begin{array}{c}\text { Examinados } \\
n ?\end{array}$} & \multicolumn{2}{|c|}{ Positivos } & \multirow{2}{*}{$\begin{array}{c}\text { Examinados } \\
\text { n? }\end{array}$} & \multicolumn{2}{|c|}{ Positivos } & \\
\hline & & n? & $\%$ & & n? & $\%$ & \\
\hline Área I & 55 & 29 & 52,7 & 78 & 39 & 50,0 & $\begin{array}{c}0,02 \\
p>0,05\end{array}$ \\
\hline Área II & 78 & 53 & 67,9 & 131 & 40 & 30,5 & $\begin{array}{c}26,22 \\
p=0,00001\end{array}$ \\
\hline Área III & 52 & 24 & 46,2 & 72 & 17 & 23,6 & $\begin{array}{c}5,95 \\
p=0,01\end{array}$ \\
\hline
\end{tabular}


Dos flebotomíneos encontrados na área I a Lutzomyia whitmani já foi descrita como vetora da Leishmania braziliensis ssp. no Brasil 14,18,19 e mesmo na Bahia"s.

Em Lençóis a L.b.braziliensis assim como a Leishmania mexicana amazonensis já foram isoladas de diversos casos humanos de leishmaniose tegumentar ${ }^{10}$. A Lutzomyia longipalpis vetora das leishmanioses viscerotrópicas da América foi a espécie mais encontrada no presente estudo. Apesar da mesma nunca haver sido descrita como vetora de leishmanias dermatotrópicas na natureza, esta espécie tem sido utilizada com frequiência em infecções experimentais em laboratórios com L.m.amazonensis e outras leishmanias de pele ${ }^{12,16}$. Isto, entretanto, não é suficiente para implicar esta espécie como vetora destas leishmanias, particularmente em Lençóis.

Não é possivel afirmar a existência do mesmo padrão de transmissão da LTA descrito para a área I nas outras áreas da cidade. Os dados do inquérito foram insuficientes para assegurar a não existência de flebotomíneos naquelas áreas, considerando o curto espaço de tempo da sua realização e a diminuição da densidade vetorial nas áreas, seja espontaneamente ou pela aplicação de inseticidas residuais nas habitações $e$ anexos, o que ocorreu em Lençóis previamente ao inquérito. A diminuição da densidade vetorial por intermédio do uso de inseticidas, já foi descrita para outras áreas do país admitindo-se, inclusive, a interrupção da transmissão da LTA $^{8,15}$.

É provável também que ocorra a transmissão silvestre da enfermidade, pois, registraram-se casos de LTA entre turistas que frequentaram os pontos de atração fora da cidade e em mora- dores de localidades situadas fora de Lençóis. Ressalta-se contudo que o padrão encontrado na área estudada é similar ao descrito em outros estudos no Brasil 7,9,21,22, sugerindo que ao longo do tempo e em várias áreas do país, vem se modificando a transmissão silvestre da LTA.

Excetuando-se a área 1 do estudo, os resultados apontam para uma associação entre as taxas de infecção de LTA e a ocupação dos individuos.

O grupo ocupacional de lavradores e garimpeiros representa grupo mais exposto à LTA por sua concentração nas áreas residenciais periféricas e por ter mais contatos com a mata em função do seu locus de trabalho. Desta forma eles estão mais expostos às duas modalidades de transmissão: a peridomiciliar e a silvestre.

Para apreciação da consistência dos resultados, ressalte-se que o grupo de lavradores e garimpeiros estudado constitui grupo relativamente homogêneo em termos de ocupação. Desta forma, alguns indivíduos participavam simultaneamente de ambas as ocupações com jornadas de trabalho maiores ora como lavrador ora como garimpeiro. Para outros indivíduos foi comum a passagem ao longo da vida profissional de garimpeiro e lavrador e vice-versa.

\section{AGRADECIMENTOS}

Ao Ministério da Saúde - Secretaria de Ciência e Tecnologia; à Diretoria Regional da SUCAM na Bahia; à Prefeitura Municipal da cidade de Lençóis, pelo apoio na realização do trabalho; ao Dr. Ítalo Sherlock, da Fundação Oswaldo Cruz, pela identificação dos flebotomíneos.

DOURADO, M.I.C. et al. [Epidemiology of leishmaniasis related to agriculture and prospecting in a locality of the State of Bahia, Brazil]. Rev. Saúde públ., S.Paulo, 23:2-8, 1989.

\begin{abstract}
This study was carried out in the city of Lençois, State of Bahia, with the objective of verifying the association between leishmaniasis infection and occupation. A Montenegro test and a questionnaire including biological and socio-economic variables were applied to the study group. Sandflies were captured in and around dwellings. The higher-than-average prevalence of leishmaniasis observed among agricultural workers and prospectors is explained by the double exposure to the infection-both at home and at work.
\end{abstract} vectors.

KEYWORDS: Leishmaniasis, mucocutaneous, occurence. Occupation. Phlebotomus. Ecology, 


\section{REFERÊNCIAS BIBLIOGRÁFICAS}

1. ARZUBIAGA, C. et al. Estudio epidemiologico de leishmaniasis tegumentar americana en Mayapuco, Peru. Bol.Ofic.sanit.panamer., 96:417-24, 1984.

2. BARRETO, A.C. et al. Caracteristicas epidemiológicas da leishmaniose tegumentar americana em uma região endêmica do Estado da Bahia. I - Leishmaniose humana. Bol.Ofic.sanit.panamer, 90:415-22, 1981.

3. BONFANTE-GARRIDO, R. Leishmanias y leishmaniasis tegumentaria en America Latina. Bol.Ofic.sanit.panamer, 95:418-26, 1983.

4. BONFANTE-GARRIDO, R. \& BARRETO, T. Leishmaniasis tegumentaria americana en el Distrito Urdaneta, Venezuela. Bol.Ofic.sanit.panamer., 91:30-1, 1981.

5. BRAY, R.S. Epidemiology of leishmaniasis: some reflections on causation. In: Trypanosomiasis and leishmaniasis with special reference to Chagas' disease. Amsterdam, Associated Scientific Publishers, 1974. p. 87.105 (CIBA Foundation Symposium, 20. New Series)

6. CAHILL, K.M. et al. A leishmanin survey in Giohar Somalia. Trans.roy.Soc.trop.Med.Hyg., 61:340-2, 1967.

7. FORATTINI, O.P. et al. Nota sobre leishmaniose tegumentar no litoral sul do Estado de São Paulo, Brasil. Rev. Saúde públ., S. Paulo, 7:447-52, 1973.

8. FORATTINI, O.P. et al. Observaçőes sobre a transmissão da leishmaniose tegumentar no Estado de São Paulo. Rev. Saude públ., S.Paulo, 10:31-43, 1976.

9. GOMES, A. de C. et al. Ecological aspects of American cutaneous leishmanisis. 4 - Observations on the endophilic behavior of the sanfly and the vectorial role of Psychodopygus intermedius in the Ribeira Valley region of the São Paulo State, Brazil. Rev. Saúde públ., S.Paulo, 20:280-7, 1986.

10. GRIMALDI Jr., G. Identificação e caracterizaçăo imunológica com anticorpos monoclonais de amostras de Leishmania do Novo Mundo e sua distribuição geográfica. Rio de Janeiro, 1987. [Tese de Doutorado - UFRJ]

11. HOCK, A. et al. Isolation of Leishmania braziliensis braziliensis and other trypanosomatids from Phlebotomine in a mucocutaneous leishmaniasis endemic area, Bahia, Brazil. Mem.Inst.Oswaldo Cruz, Rio de Janeiro, 81 (Supl.):62, nov., 1986.

12. KILLICK-KENDRICK. Biology of Leishmania in phlebotomine sandflies. In: Lumsden, W.H.R. \& Evans, D.A., eds. Bıology of the Kineloplastida. New York, Academic Press, 1979. p. 395-460.
13. LAISON, R. Epidemiologia e ecologia da lejshmaniose tegumentar na Amazônia. Hiléia méd., Belém, 3(1): $135-40,1981$.

14. LAISON, R. \& SHAW, J.J. The role of animals in the epidemiology of South American Leishmaniasis. In: Lumsden, W.H.R. \& Evans, D.A., eds. Biology of the Kinetoplastida. New York, Academic Press, 1979. p.1-116.

15. LIMA, L.C. et al. Observaçōes sobre a leishmaniose tegumentar cinco anos após profilaxia. Rev. Saúde públ., S.Paulo, 22:73-7, 1988.

16. MOLYNEUX, D.H. et al. Leishmania in phlebotomine sandflies. III - The ultrastructure of Leishmania in phlebotomine sandflies. Proc.roy. Soc.Lond.B., 190:341-57, 1975.

17. MOVSKOVSIJ, S.D. \& DUNA NINA, N.N. Epidemiology of the leishmaniasis, general considerations. Bull.Wld Hlth Org., 44:529-34, 1971.

18. ORGANIZACION MUNDIAL DE LA SALUD. Comite de Expertos en Leishmaniasis, Ginebra, 1982. Informe. Ginebra, 1984. (Série de Informes Técnicos, 701).

19. PESSOA, S.B. \& COUTINHO, J.O. Infecção natural do Phlebotomus pessoai por faunas em leptomonas provavelmente da Leishmania braziliensis. Rev.Biol.Hig., S.Paulo, 10:139-42, 1940.

20. PESSOA, S.B. Endemias parasitárias da zona rural brasileira. São Paulo, 1963. p. 376-433.

21. PROENÇA, N.G. \& MULLER, H. Nota sobre a ocorrência da leishmaniose tegumentar na Serra da Cantareira, Såo Paulo, Brasil. Rev. Saúde públ., S.Paulo, 13:60-2, 1979.

22. ROCHA e SILVA, E.O. et al. A leishmaniose tegumentar americana no litoral sul do Estado de São Paulo. Rev. bras. Malar., 32:9-25, 1980.

23. SALGADO, V. Aspectos epidemiológicos da leishmaniose tegumentar na Região Amazonica. An.bras.Derm., 57:141-4, 1982.

24. SECRETARIA DA FAZENDA DA BAHIA. Catálogo de informaçð̃es sócio-econômicas dos municipios. Salvador, Escola de Administraçăo Fazendária, 1984.

25. SUPERINTENDENCIA DE CAMPANHAS DE SAÚDE PÚBLICA (SUCAM). Diretoria Regional da Bahia. Relatorio da campanha de controle das leishmanioses. Salvador, 1968. [Mimeografado].

Recebido para publicaçđo em 13/10/1987 Reapresentado em $9 / 8 / 1988$ Aprovado para publicaçâo em 19/9/1988 